BULL. AUSTRAL. MATH. SOC.

VOL. 34 (1986) $127-132$.

\title{
EVENTUALLY REGULAR SEMIGROUPS \\ THAT ARE GROUP-BOUND
}

\author{
P.M. EDWARDS
}

\begin{abstract}
Necessary and sufficient conditions are given for certain classes of eventually regular semigroups to be group-bound or even periodic.
\end{abstract}

\section{Introduction and Preliminaries.}

Wherever possible the notation and conventions of Clifford and Preston $[1,2]$ will be used.

An element of a semigroup $S$ is called group-bound if it has some power that is in a subgroup of $S$ and is called eventually regular if it has some power that is regular. A semigroup $S$ is group-bound [eventually regular] if all of its elements are group-bound [eventually regular]. Thus the class of eventually regular semigroups includes all regular semigroups and all group-bound semigroups. For more properties of eventually regular semigroups see [3].

It is of interest to know whether a semigroup under consideration is group-bound. For example it is well known that $D=J$ for a groupbound semigroup. This follows for instance from the conjuction of [6, Theorem 1.2 (vi)] and [6, Remark 1.7]. There exist regular semigroups for which $D \neq J$. In section 2 necessary and sufficient conditions for certain classes of eventually regular semigroups to be group-bound or

Received 10 October 1985. The author wishes to thank the referees for remarks concerning the paper's presentation.

Copyright Clearance Centre, Inc. Serial-fee code: 0004-9727/86 $\$ A 2.00+0.00$. 
even periodic are given whence $D=J$ for these semigroups.

Recall from [3] or [4] the idempotent-separating congruence on a semigroup $S, \mu=\mu(S)$ defined by,

$\mu=\{(a, b) \in S \times S \mid$ if $x \in S$ is regular then each of $x R x a$, $x R x b$ implies $x a y b$ and each of $x<a x, x<b x$ implies $a x H b x\}$.

The congruence $\mu$ is the maximum idempotent-separating congruence on any eventually regular semigroup [3, Theorem 11]. In this paper we use the generalized meaning of fundamental given in [4]; thus a semigroup $S$ is called fundomental if the only idempotent-separating congruence on $S$ is ${ }^{1} S$. Alternatively, by a result of D. Easdown, we may view a semigroup $S$ as fundamental if and only if $\mu(S)={ }^{1} S$. Since $\mu$ is the identity relation on $S / \mu[4$, Theorem 8$]$ it follows that $S / \mu$ is always fundamental.

2. Eventually Regular Semigroups that are Group-Bound.

Theorems 3, 4 and 6 indicate when certain classes of eventually regular semigroups are group-bound or even periodic. Theorem 5 [Theorem 7] indicates when certain classes of eventually regular semigroups have the property that for each member $S$ the semigroup $S / \mu$ is periodic [finite]; thus any member of such a class if fundamental is periodic [finite].

LEMMA 1. If the H-class of $a^{k}, H$ say, is a group then $a^{n} \in H$ for aZz $n \geq k$.

Proof. Suppose the identity element of $H$ is $e$ and let $y$ be the group inverse of $a^{k}$ in $H$. It follows easily that $e a=a e$ and that $a y=a e y=e a y=y a^{k} a y=y a a^{k} y=y a e=y e a=y a$. Let $r$ be an integer such that $k r>n$ and let $v=a^{k r-n} y^{r}$. Then since $a y=y a$, $v a^{n}=a^{n} v=a^{n} a^{k r-n} y^{r}=a^{k r} y^{r}=\left(a^{k} y\right)^{r}=e^{r}=e$. Because $n \geq k$ it is clear that $e a^{n}=a^{n} e=a^{n}$. Thus $a^{n} \in H_{e}=H$ as required.

COROLLARY 2. If $a^{k} \mathrm{H} a^{k+r}$ with $r>0$, then the H-class of $a^{k}$ is a group. 
Proof. Choose $p \geqq 0$ such that $r$ divides $k+p$ with say $r q=k+p$. Then since $a^{k} H a^{k+r}$ it follows easily that $a^{k} H a^{k+r i}$ for all $i \geq 1$. In particular when $i=q, a^{k} H a^{k+r q}$ and so also $a^{k+p} H a^{k+r q+p}$, whence $a^{r q} H a^{2 r q}$. It follows from Green's theorem that the $H$-class of $a^{r q}$ is a group. From Lemma $1, a^{n} H a^{r q}$ for all $n \geqq r q$ and so $a^{k} H a^{k+r q} H a^{r q}$ whence the $H$-class of $a^{k}$ is a group.

THEOREM 3. Let $S$ be on eventualiy regular semigroup such that each regular D-class of $S$ contains at most $m$ L-classes of $S$, for some integer $m$. Take any $a \in S$ and let $k$ be the least integer greater than or equal to $m$ such that $a^{k}$ is regular. Then there exists a subgroup $H$ of $S$ such that $a^{n} \in H$ for all $n \geqq k$. In particular $S$ is group-bound.

Proof. Let $S, m$, and $k$ be as stated in the theorem. The integer $k$ exists since for example $\left(a^{m}\right)^{p}$ is regular for some $p \geqq 1$. since $a^{k}$ is regular there is an idempotent $e$ such that $e R a^{k}$. For all $0 \leqq i \leqq k, e a^{i}\left(a^{k-i}\right)=a^{k}$ and $a^{k} u a^{i}=e a^{i}$ where $u$ is such that $a^{k} u=e$, whence $e a^{i} R a^{k}$. By definition of $m$, and because $k \geq m$, there exist $0 \leqq s<t \leqq k$ such that $e a^{s}=e a^{t}$. Since $L$ is a right congruence, it follows that $e a^{k}=a^{k} L e a^{k+t-s}$ and that $e a^{k-t+s} L e a^{k}=a^{k}$. Because $0<t-s \leqq k, e a^{k-t+s} R a^{k}$ follows from above. Since $e a^{k-t+s} R a^{k}$ and $a^{k}$ is equal to the right translation of $e a^{k-t+s}$ by $a^{t-s}$ and $a^{k} \in L_{e} a^{k-t+s}$, Green's lemma yields that $a^{k} R a^{k} a^{t-s}$ whence $a^{k} R a^{k+t-s}$. It is now clear that $a^{k} H a^{k+t-s}$. It follows that the H-class of $a^{k}$ is a group by Corollary 2 and so $a^{n} H a^{k}$ for all $n \geqq k$ by Lemma 1 .

When applied to regular semigroups Theorem 3 reduces to Theorem 15 of Hall [5]. The next result gives a condition that is necessary and 
sufficient for a certain class of eventually regular semigroups to be group-bound.

THEOREM 4. Let $S$ be an eventually regular semigroup with only finitely many regular D-classes. Then $S$ is group-bound if and only if $S$ contains no copy of the bicyclic semigroup.

Proof. Necessity is clear since the bicyclic semigroup is not group-bound and any eventually regular subsemigroup of a group-bound semigroup is group-bound. Suppose that $S$ contains no copy of the bicyclic semigroup. Thus no pair of distinct comparable idempotents are D-related. Take $a \in S$. Since there are only finitely many regular $D$-classes and infinitely many powers of $a$ are regular it follows that there exists distinct positive integers $m$ and $n$ such that $a^{m} D a^{n}$ with $a^{m}$ regular. Since $L_{a}{ }_{a}$ and $L_{a}{ }^{n}$ are regular $L$-classes there exist idempotents $e \in L_{a}^{m}$ and $f \in L_{a}{ }^{n}$. Without loss of generality $n<m$ and so $L_{a}^{m} \leqq L_{a}{ }^{n}$, whence $e f=e$. Putting $g=f e$ yields $g=g^{2}$, $g \leqq f$ and $g L_{e} D f$, whence $g=f$ and so $L_{a^{n}}=L_{a^{m}}$. Dually, $R_{a^{n}}=R_{a}^{m}$ and so $a^{n} H_{a^{m}}$, whence from corollary 2, $S$ is group-bound.

THEOREM 5. Let $S$ be con eventually regular semigroup and let $m$ be an integer such that each regular D-class of $S$ contains at most $m$ idempotents. Then $S / \mu$ is periodic. Thus if $S$ is fundomental then $S$ is periodic.

Proof. The following representation of an arbitrary semigroup $T$ will be used. Let $X$ be the set of regular L-classes of $T$ and let $Y$ be the set of regular R-classes of $T$. Define $\phi: T \rightarrow P T_{X} \times P T_{Y}^{*}$ by $s \phi=\left(\rho_{s}, \lambda_{s}\right)$, where $\rho_{s}: L_{x} \rightarrow L_{x s}$ if $x$ is regular and $x R_{x s}$ and is undefined otherwise and $\lambda_{s}: R_{x} \rightarrow R_{s x}$ if $x$ is regular and $x$ Ls $x$ and is undefined otherwise. Here $P T_{Y}^{*}$ denotes the dual of the semigroup $P T_{Y}$. Then $\phi$ is a representation of $T$ and $\mu(T)=\operatorname{ker} \phi \quad$ (see [3, page 30]). Now put $T=S, Z=\{1,2, \ldots, m\}$ and let $n$ be an integer 
such that $b^{n}$ is idempotent for each $b$ in $P T_{Z}$. Take $c \in S$, choose $k \geqq 1$ such that $\left(c^{m}\right)^{k}$ is regular and put $a=e^{k}$. It will suffice to show that $a \mu$ is periodic in $S / \mu$. Note that $a^{m}$ is regular.

Let $D$ be any regular $D$-class of $S$ and let $D / L$ denote the (finite) set of $L$-classes contained in $D$. Using the method of Hall [5, Theorem 15] it can be shown that $\rho_{a}^{m}$ maps $(D / L) \cap$ range $\rho_{a}^{m}$ one to one onto itself. It follows that $\rho_{a}^{m} H \rho_{a}^{n} H \rho_{a}^{n m}$ in $P T_{X}$. Put $z_{D}=(D / L) n$ range $\rho_{a}^{m}$. Since $\left|z_{D}\right| \leqq m,\left(\rho_{a}^{m}\right)^{n}$ maps $z_{D}$ identically to itself, by the choice of $n$. Thus $\rho_{a}^{m m}$ maps $\cup\left\{z_{D} \mid D \in D\right\}=$ range $\rho_{\alpha}^{m m}$ identically to itself, whence $\rho_{\alpha}^{m n}$ is an idempotent of $P T_{X}$. Dually, $\lambda_{a}^{m n}$ is an idempotent of PT ${ }_{Y}^{*}$, whence $\left(a^{m n}, a^{2 m n}\right) \in$ ker $\phi=\mu$ and so $a^{m n}$ is idempotent in $S / \mu$.

Remark 1. [5, page 19] In general a fundamental regular semigroup in which each $D$-class contains one L-class is not necessarily periodic. As an example let $G$ be a non-periodic group, let $G^{\prime}=\left\{g^{\prime} \mid g \in G\right\}$ be a disjoint copy of $G$ and put $S=G \cup G^{\prime}$. Extend the multiplication from $G$ to $S$ be defining, for all $g, g_{1}, g_{2} \in G, g_{1}^{\prime} g=g_{1}^{\prime} g_{2}^{\prime}=g_{1}^{\prime}$ and $g g_{1}^{\prime}=\left(g g_{1}\right)^{\prime}$. Then $S$ is a fundamental semigroup, $D=L$ and $S$ is of course not periodic.

THEOREM 6. Let $S$ be on eventualiy regular semigroup with only finitely many regular elements. Then $S$ is periodic.

Proof. Take any $a \in S$. Then $a^{k}$ is regular for some $k_{1}>1$ as for example $k_{1}$ could be $2 k_{0}$ where $k_{0}$ is an integer such that $\left(a^{2}\right)^{k_{0}}$ is regular. Similarly there exists $k_{2}>1$ such that $\left(a^{k_{1}}\right)^{k_{2}}$ is regular and $k_{3}>1$ such that $\left(a^{k_{1} k_{2}}\right)^{k_{3}}$ is regular. Now 
$k_{1}<k_{1} k_{2}<k_{1} k_{2} k_{3}$ and by continuing this process it is clear that there exist distinct integers $n$ and $m$ such that $a^{n}=a^{m}$. Thus $a$ is periodic, whence $S$ is periodic.

As a final specialization we have the following theorem:

THEOREM 7. [3, Theorem 15]. Let $S$ be cony semigroup with only finitely many idempotents. Then $S / \mu$ is finite. Thus if $S$ is fundomental then $S$ is finite.

\section{References}

[1] A. H. Clifford and G. B. Preston, The algebraic theory of semigroups, Vol. I. (Mathematical Surveys, 7. Amer. Math. Soc., Providence, R. I. 1961).

[2] A. H. Clifford and G. B. Preston, The algebraic theory of semigroups, Vol. II. (Mathematical Surveys, 7. Amer. Math. Soc., Providence, R.I., 1967).

[3] P. M. Edwards, "Eventually regular semigroups," Bulz. Austral. Math. Soc. 28 (1983), 23-38.

[4] P. M. Edwards, "Fundamental semigroups," Proc. Roy. Soc.Edinburgh Sect. A 99 (1985), 313-317,

[5] T. E. Hall, "On regular semigroups," J. Algebra 24 (1973), 1-24.

[6] T. E. Ha1l and W. D. Munn, "Semigroups satisfying minimal conditions II," GLasgow Math. J. 20 (1979), 133-140.

Department of Econometrics

Monash University

clayton

Victoria 3168

Australia. 